\title{
Mathematical Modeling of Spherical Microstrip Antennas and Applications
}

\author{
Nikolaos L. Tsitsas and Constantinos A. Valagiannopoulos \\ National Technical University of Athens \\ Greece
}

\section{Introduction}

Microstrip antennas offer many attractive features and hence it is very challenging to model efficiently their resonance and radiation characteristics (Pozar \& Schaubert, 1995). In particular, microstrip antennas composed of perfectly electric conducting (PEC) patches mounted on spherical surfaces possess two basic advantages against the planar ones. First, their curved shape makes them more applicable to real-world moving configurations such as missiles, satellites and aircrafts, where large plane boundaries are usually absent. Second, the frequency scanning problem encountered in case of planar microstrips at low elevations may be overcome due to the conformability of spherical antennas. Apart from these two advantages, spherical microstrip antennas offer (due to their relatively simple geometry) additional attractive features like low cost, light weight, easy fabrication as well as integrability with microwave and millimeter-wave circuits. As far as the metallic patch is concerned, circular or annular-ring ones are preferable for mounting on a spherical body. Interesting design characteristics of microstrip antennas are mainly the complex resonant frequencies, the radiation pattern and the input impedance. An extensive literature survey concerning the analysis of non-planar radiating microstrip structures, as well as the investigations of the aforementioned design characteristics is included in the classic book (Wong, 1999).

The mathematical methods, developed for analyzing the behavior of a spherical microstrip, may be categorized as follows: (i) the full-wave approach implements the method of moments (MoM) to approximate the surface current on the PEC patch by means of suitable basis functions (Uwaro \& Itoh, 1989; Tam \& Luk, 1991; Wong, 1999, Sipus et al., 2003; Giang et al. 2005), (ii) the cavity model analysis assumes that the distance between the substrate and the radiating metallic patch is electrically small, forming a cavity into which the vector of the electric field is independent of the longitudinal coordinate (Lo et al., 1979; Luk \& Tam 1991; Chen \& Wong, 1994), (iii) the generalized transmission-line model (GTLM) extracts an equivalent circuit for the spherical microstrip, where the line parameters are the electromagnetic fields under the patch (Ke \& Kishk, 1991; Kishk, 1993).

Regarding the analysis of spherical microstrips, whose circular metallic patch is located inside a coating, composed of an arbitrary number of concentric spherical substrate and superstrate layers, an efficient approach for the analysis of the related resonance and radiation characteristics has been proposed in (Valagiannopoulos \& Tsitsas, 2008a; Valagiannopoulos \& Tsitsas, 2008b). The generic structure of that microstrip configuration 
offers in particular additional degrees of freedom to the designer in order to achieve the required operational characteristics. More precisely the method, presented in (Valagiannopoulos \& Tsitsas, 2008a), utilizes appropriately the Legendre transform, since the microstrip structure is non-entire with respect to the spherical coordinates variable $\theta$, while the effect of the layers above and below the patch is handled efficiently by using a T-matrix method (Chew, 1995; Tsitsas \& Athanasiadis, 2006). Suitable basis functions for describing the surface current on the patch are chosen by applying the cavity model analysis (Lo et al., 1979). Subsequently, Galerkin's method is employed to formulate a homogeneous linear system, the singular points of which are the complex resonant frequencies. Finally, the inverse Legendre transform and far-field asymptotics lead to the computation of the radiation patterns. Importantly, structures, involving microstrips with a fixed small number of substrate and superstrate layers (Tam \& Luk, 1991; Wong et al., 1993a; Wong \& Chen, 1993), are incorporated as special cases of this method.

Several spherical microstrip configurations have been reported in the literature over the past two decades, exhibiting various interesting radiation features and demonstrating useful design characteristics. For example, the resonance properties of a simple spherical microstrip, possessing a single dielectric substrate between the metallic sphere and the circular patch are investigated in (Tam \& Luk 1991). Moreover, in (Wong et al., 1993a) the authors studied the influence of a dielectric superstrate on the resonance and radiation properties of the spherical circular patch antenna proposed in (Tam \& Luk 1991). A spherical microstrip with a substrate of two layers, one of which is an airgap, was treated in (Wong \& Chen, 1993). In a similar context, a spherical microstrip with an annular-ring patch and an air gap between the metallic sphere and the dielectric was treated in (Ribero et al., 1999). On the other hand, a 4-layered microstrip with two airgaps surrounding an amplifying layer (constituting actually a $\varphi$ - and $\theta$-entire excitation) was proposed in (Valagiannopoulos \& Tsitsas, 2008a), where the control mechanism of the amplifying capability via the layer's thickness and dielectric permittivity was also reported. Moreover, a spherical microstrip with two airgaps, surrounded by a zero-index material, expected to find applications in receiving antennas, was proposed in (Valagiannopoulos \& Tsitsas, 2008a). On the other hand, a coating's continuous distribution, following a "shifted" Luneburg law may be treated by a step approximation of the radial function of its dielectric permittivity (Nikolic et al., 2007; Sakurai et al. 1998; Liang et al. 2005; Valagiannopoulos \& Tsitsas, 2008a). Such a configuration exhibits larger quality factor values than respective single-layered spherical microstrips, which especially become extremely large as the patch approaches the metallic sphere (Valagiannopoulos \& Tsitsas, 2008a). Besides, a PEC patch (implant) radiating inside a 6-layered spherical human head model was investigated in (Kim \& Rahmat-Samii, 2004).

The present chapter is organized as follows. Section 2 contains an overview of existing mathematical methods, mainly treating single-layered spherical microstrip antennas. Section 3 discusses the motivation to model multi-layered spherical microstrips, where the patch is arbitrarily located inside the multi-layered sphere, and summarizes the basic features of the combined Legendre transform and T-matrix methodology for the analysis of such microstrip configurations. Furthermore, in Section 4 potential applications of multilayered spherical microstrips are pointed out, dealing with coating's materials characterized by (i) zero refractive index, (ii) amplifying properties, and (iii) continuous dielectric permittivity distribution. 


\section{Overview of mathematical methods treating single-layered microstrips}

In this Section we give a brief report of the existing mathematical methodologies, which provide the essential tools for the analysis of single-layered spherical microstrips. Hereinafter, an $\exp (+j \omega t)$ time dependence of the field quantities is assumed and suppressed.

\subsection{Full-wave approach}

In full wave analysis, one seeks the unique field solution satisfying the Helmholtz differential equation as well as the appropriate boundary conditions (see Wong, 1999 for an excellent survey on full wave methods). Into this context, we consider the microstrip configuration of Fig. 1, where a PEC sphere of radius $a_{1}$ is coated within a dielectric substrate of thickness $d$. A circular PEC radiating patch, located at $r=a_{2}$ and $\theta<\theta_{p}$ and assuming to possess negligible thickness, is mounted on the dielectric substrate. In order to fulfill the boundary conditions, the tangential electric field is required to be equal to zero on the PEC patch.

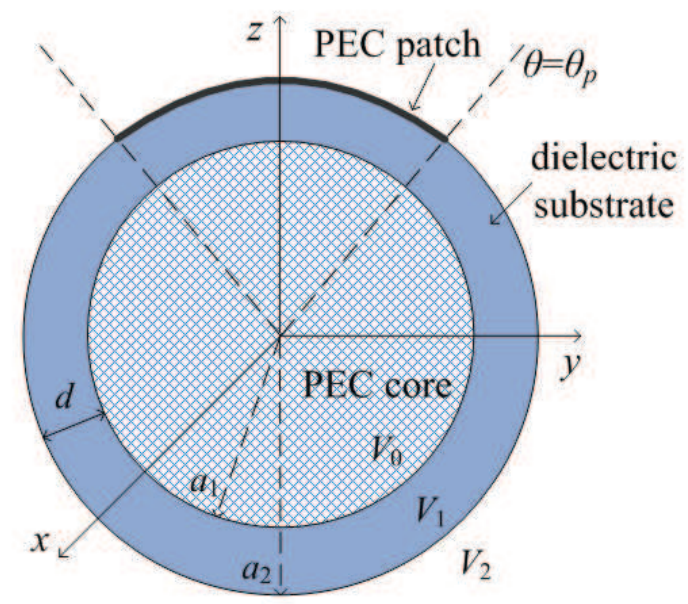

Fig. 1. Geometry of the spherical microstrip composed of a PEC sphere of radius $a_{1}$ coated with a dielectric substrate of thickness $d$; the circular PEC patch is located at $r=a_{2}$ and $\theta<\theta_{p}$.

The solution of the radiation problem is actually reduced to determining the surface current distribution on the patch. Since the patch is not entire with respect to the inclination angle $\theta$ (although it is entire with respect to the azimuthal angle $\varphi$ ), it is convenient to utilize the vector Legendre Transform pair, defined in (Tam \& Luk, 1991; Wong, 1999; Giang et al. 2005) by

$$
\begin{gathered}
\mathbf{F}(r, \theta)=\sum_{n=|m|}^{+\infty} \overline{\overline{\mathbf{L}}}(n, m, \theta) \cdot \tilde{\mathbf{F}}(r, n), \\
\tilde{\mathbf{F}}(r, n)=\frac{1}{S(n, m)} \int_{0}^{\pi} \overline{\overline{\mathbf{L}}}(n, m, \theta) \cdot \mathbf{F}(r, \theta) \sin \theta d \theta,
\end{gathered}
$$


where $\tilde{\mathbf{F}}(r, n)$ constitutes the Legendre Transform of $\mathbf{F}(r, \theta)$. Both functions are $2 \times 1$ vectors, containing the transverse (with respect to $\hat{\mathbf{r}}$ ) components of either the electric or the magnetic field or the surface current, namely $\mathbf{F}(r, \theta)=\left[\mathbf{F}_{\theta}(r, \theta), \mathbf{F}_{\varphi}(r, \theta)\right]^{T}$ and $\tilde{\mathbf{F}}(r, n)=\left[\tilde{\mathbf{F}}_{\theta}(r, n), \tilde{\mathbf{F}}_{\varphi}(r, n)\right]^{T}$. The remaining participating quantities in (1a) and (1b) are given by

$$
\begin{aligned}
& \overline{\overline{\mathbf{L}}}(n, m, \theta)=\left[\begin{array}{cc}
\frac{\partial P_{n}^{m}(\cos \theta)}{\partial \theta} & -j m \frac{P_{n}^{m}(\cos \theta)}{\sin \theta} \\
j m \frac{P_{n}^{m}(\cos \theta)}{\sin \theta} & \frac{\partial P_{n}^{m}(\cos \theta)}{\partial \theta}
\end{array}\right], \\
& S(n, m)=\frac{2 n(n+1)(n+m) !}{(2 n+1)(n-m) !} .
\end{aligned}
$$

Note that the use of the vector Legendre Transform in the spherical microstrip is the analog situation to the utilization of the Fourier (Tsalamengas et al., 1985; Wong et al., 1993b) and the Hankel (Ali et al., 1982; Nie et al., 1990) transform in the planar one.

The electric and magnetic fields $\mathbf{E}^{1}$ and $\mathbf{H}^{1}$ in the substrate region $V_{1}$ as well as the respective ones $\mathbf{E}^{2}$ and $\mathbf{H}^{2}$ in the outer (air) region $V_{2}$ are expressed by means of the corresponding electric and magnetic potentials. Subsequently, these potentials are expanded with respect to an appropriate basis of spherical harmonic functions. The unknown weighting coefficients in these expansions will be determined by imposing the appropriate boundary conditions.

To this end, the vector Legendre Transform is utilized in order to overcome the difficulty concerning the limited $\theta$ extent of the patch. Specifically, the boundary conditions are transformed from the spatial into the spectral (Legendre) domain as

$$
\begin{gathered}
\tilde{\mathbf{E}}^{1}(r, n)=\left\{\begin{array}{cc}
0, & r=a_{1}, \\
\tilde{\mathbf{E}}^{2}(r, n), & r=a_{2}
\end{array}\right. \\
\tilde{\mathbf{J}}_{s}\left(a_{2}, n\right)=\left[\begin{array}{cc}
0 & -1 \\
1 & 0
\end{array}\right]\left[\tilde{\mathbf{H}}^{2}\left(a_{2}, n\right)-\tilde{\mathbf{H}}^{1}\left(a_{2}, n\right)\right],
\end{gathered}
$$

where $\tilde{\mathbf{J}}_{s}\left(a_{2}, n\right)$ is the spectral amplitude of the surface current density on the circular patch. By transforming the electric and magnetic field expressions in the spectral domain (resulting by considering the corresponding expansions of the potentials, as described above) and utilizing the transformed boundary conditions ( $3 a)$ and ( $3 b)$, the following spectral equation, connecting the surface current density with the tangential electric field on the patch, is derived

$$
\tilde{\mathbf{E}}\left(a_{2}, n\right)=\overline{\overline{\mathbf{Z}}}(n) \cdot \tilde{\mathbf{J}}_{s}\left(a_{2}, n\right)
$$

where $\overline{\overline{\mathbf{Z}}}(n)$ is a determined "impedance" matrix, while $\tilde{\mathbf{E}}\left(a_{2}, n\right)$ denotes the common value of the tangential electric fields $\tilde{\mathbf{E}}^{1}(r, n)$ and $\tilde{\mathbf{E}}^{2}(r, n)$ on $r=a_{2}$ (see the boundary condition (3a)). 
Next, the current distribution on the PEC patch is expanded into a suitable set of orthogonal functions and the unknown weighting coefficients of these expansions are finally determined by using a Galerkin's moment method. More precisely, the surface current distribution is expressed by the following weighted finite sum of suitably chosen basis functions, as

$$
\tilde{\mathbf{J}}_{s}\left(a_{2}, n\right)=\sum_{v=1}^{\mathrm{N}} c_{v} \tilde{\mathbf{J}}_{s, v}\left(a_{2}, n\right)
$$

where $c_{v}$ the unknown coefficients and $\tilde{\mathbf{J}}_{s, v}\left(a_{2}, n\right)$ the spectral amplitude of the $v$-th expansion function. Then, by applying a Galerkin scheme, namely utilizing as testing functions the same set of basis (expansion) functions, the following homogeneous linear system is formulated

$$
\overline{\overline{\mathbf{A}}}(\omega) \cdot \mathbf{c}=\mathbf{0},
$$

where the vector $\mathbf{c}$ incorporates the coefficients $c_{v}$, while the elements of matrix $\overline{\overline{\mathbf{A}}}$ are given by (the * denotes the complex conjugate transpose)

$$
A_{\mu \nu}(\omega)=\sum_{n=|m|}^{+\infty} S(n, m) \tilde{\mathbf{J}}_{s, \mu}^{*}\left(a_{2}, n\right) \cdot \overline{\overline{\mathbf{Z}}}(n) \cdot \tilde{\mathbf{J}}_{s, v}\left(a_{2}, n\right) \quad(\mu, v=1, \ldots, \mathrm{N}) .
$$

In view of (6), the complex resonant frequencies $\omega_{0}=\operatorname{Re}\left(\omega_{0}\right)+j \operatorname{Im}\left(\omega_{0}\right)$ of the spherical microstrip structure are calculated by solving numerically the equation

$$
\operatorname{det}\left[\overline{\overline{\mathbf{A}}}\left(\omega_{0}\right)\right]=0 \text {. }
$$

Note that one should retain only the roots of (8) with $\operatorname{Re}\left(\omega_{0}\right)>0$ and $\operatorname{Im}\left(\omega_{0}\right)>0$ in order to avoid unbounded oscillations as time advances. In particular, $\operatorname{Re}\left(\omega_{0}\right)$ is the resonant frequency of the spherical microstrip and $\operatorname{Im}\left(\omega_{0}\right)$ expresses the radiation loss, defining the quality factor $Q$ of the structure by

$$
Q=\frac{\operatorname{Re}\left(\omega_{0}\right)}{2 \operatorname{Im}\left(\omega_{0}\right)}
$$

\subsection{Cavity model analysis}

The appropriate basis functions for the expansion of the surface current on the patch are selected according to the cavity model theory (Lo et al., 1979; Luk \& Tam 1991; Chen \& Wong, 1994). The basic requirements for these basis functions concern the facts that they should be orthogonal and also be transformed into the spectral domain into closed form (Wong, 1999). In particular, the cavity model theory dictates that the region between the patch and the PEC core forms a cavity which is bounded by a magnetic wall along the edge of the patch and by electric walls from above and below, and that the field in the cavity is independent of $r$ (Wong, 1999).

Subsequently, the Helmholtz equations for the electric and magnetic potentials are solved locally in an area just enclosing the patch, on which flows the unknown current. Because the 
patch is non-entire with respect to $\theta$ and due to the principle of charge preservation (vanishing current component normal to the edge of the metallic patch), only specific degrees of the Legendre functions provide solutions with physical meaning. More specifically, these suitable degrees for the $\mathrm{TM}^{m \ell}$ and $\mathrm{TE}^{m \ell}$ cases are the roots $\ell^{\mathrm{TM}}(i)$ and $\ell^{T E}(i)$ respectively of the equations

$$
P_{\ell^{T M}(i)}^{\prime m}\left(\cos \theta_{0}\right)=0 \quad \text { and } \quad P_{\ell^{T E}(i)}^{m}\left(\cos \theta_{0}\right)=0
$$

where the prime indicates differentiation with respect to $\theta$ and $i$ is an integer denoting the $i^{\text {th }}$ root of the corresponding equation. The roots of (10) lie on the real axis (Abramowitz \& Stegun, 1972). Each value of $\ell^{T M}(i)$ and $\ell^{T E}(i)$ corresponds to a specific operation TM ${ }^{m}$ and $\mathrm{TE}^{m \ell}$ mode. Furthermore, by imposing the discontinuity boundary condition for the magnetic field, one obtains the explicit forms of the current basis functions for the $\mathrm{TM}^{m} \mathrm{l}$ and TE ${ }^{m \ell}$ modes

$$
\begin{aligned}
& \mathbf{J}_{s}^{T M_{m \ell(i)}}(\theta)=\left\{\begin{array}{cc}
{\left[\begin{array}{c}
P_{\ell^{T M}(i)}^{\prime m}(\cos \theta) \\
\frac{j m}{\sin \theta} P_{\ell^{T M}}^{m}(i) \\
\mathbf{0}
\end{array}\right], \quad \theta<\theta_{p},} & \theta>\theta_{p}
\end{array},\right. \\
& \mathbf{J}_{s}^{T E_{m \ell(i)}}(\theta)=\left\{\begin{array}{cc}
{\left[\frac{j m}{\sin \theta} P_{\ell^{T E}}^{m}(i)\right.} \\
-P_{\ell^{T E}(i)}^{\prime m}(\cos \theta) \\
\mathbf{0}, & \theta<\theta_{p} .
\end{array} .\right.
\end{aligned}
$$

Both current basis functions are zero for $\theta_{p}<\theta<\pi$, since the patch there is absent.

Importantly, the elements $A_{\mu v}(\omega)$ of (7) involve the current basis functions in the spectral (Legendre) domain, which are derived by imposing the Legendre transformations on the currents (11), yielding

$$
\begin{aligned}
& \tilde{\mathbf{J}}_{s}^{T M_{m \ell(i)}}(n)=\frac{1}{S(n, m)}\left[\begin{array}{c}
\frac{\ell^{T M}(i)\left(\ell^{T M}(i)+1\right)}{\ell^{T M}(i)\left(\ell^{T M}(i)+1\right)-n(n+1)} P_{\ell^{T M}(i)}^{m}\left(\cos \theta_{p}\right) P_{n}^{\prime m}\left(\cos \theta_{p}\right) \sin \theta_{p} \\
j m P_{\ell^{T M}(i)}^{m}\left(\cos \theta_{p}\right) P_{n}^{m}\left(\cos \theta_{p}\right)
\end{array}\right], \\
& \tilde{\mathbf{J}}_{s}^{T E_{m \ell(i)}}(n)=\frac{1}{S(n, m)}\left[\begin{array}{c}
0 \\
\frac{n(n+1)}{\ell^{T E}(i)\left(\ell^{T E}(i)+1\right)-n(n+1)} P_{\ell^{T E}(i)}^{\prime m}\left(\cos \theta_{p}\right) P_{n}^{m}\left(\cos \theta_{p}\right) \sin \theta_{p}
\end{array}\right] .
\end{aligned}
$$

\subsection{Generalized transmission line model}

The transmission-line model (TLM) was one of the first and simplest methods for the analysis of microstrip antennas with relatively fair accuracy compared to other more complicated methods (Pues \& Van de Capelle, 1984). The TLM method in its original form 
incorporates as line parameters the characteristic impedance and the effective propagation constant and is applicable only for planar rectangular or square microstrip antennas (Wong, 1999). To this end, the generalized transmission line model (GTLM) theory was proposed (Bhattacharyya \& Garg, 1985), where the line parameters are the electromagnetic fields under the patch. Under this consideration, as long as the separation of variables is feasible for the wave equation expressed in a particular coordinate system, GTLM theory is applicable to microstrip antennas of any patch shape.

Focusing on the modeling of spherical microstrips by the GTLM theory, the patch is considered as a transmission line in the $\theta$-direction, which is loaded with a wall admittance evaluated at the radiation apertures (Ke \& Kishk, 1991; Kishk, 1993). Besides, the effect of other apertures is considered as leakage of the transmission line. The equivalent transmission line of the circular patch can be replaced by a П-network, while the wall admittance at the patch edge is considered constant. Fig. 2 depicts the equivalent circuit of the spherical microstrip with a circular patch of Fig. 1 and a probe feeding at $\theta_{f}$ and $\varphi_{f}$ (Wong, 1999). The network of the circuit elements, $g_{1}, g_{2}$, and $g_{3}$, represents the transmissionline section between the feeding and the radiation aperture at the patch edge. The shorted transmission-line section between the feeding and the patch center is replaced by an equivalent admittance $y_{f}$.

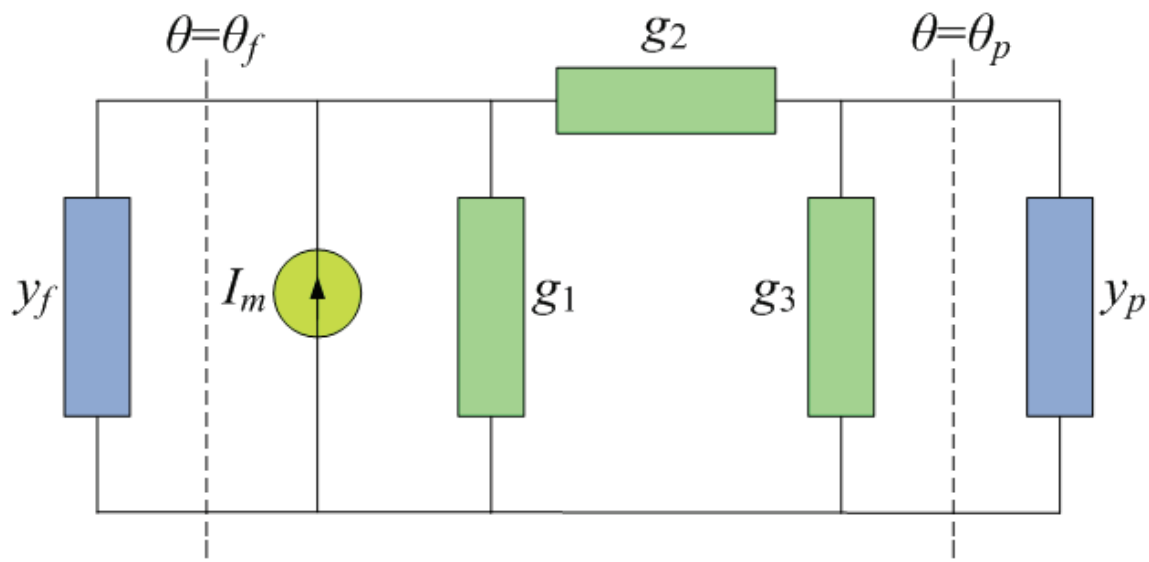

Fig. 2. Equivalent GTLM circuit for the spherical microstrip with circular patch of angular extent $\theta_{p}$ (depicted in Fig. 1), which is subject to probe feeding at $\theta_{f}$ and $\varphi_{f}$.

By deriving expressions for the corresponding circuit elements, the input impedance of the patch antenna at the feed position is readily obtained. More precisely, by following the GTLM formulation (Ke \& Kishk, 1991; Kishk, 1993), the microstrip antenna is modeled as a transmission line in the $\theta$-direction, while the modal voltage and the modal current are, respectively, defined by $E_{r m}$, and $\pm a_{1} \sin \theta H_{\varphi m}$ for wave propagation in the $\mp \hat{\boldsymbol{\theta}}$ direction, where $E_{r m}$, and $H_{\varphi m}$ are the electric and magnetic fields inside the substrate layer under the patch (Wong, 1999), which for the $\mathrm{TM}_{m n}$ mode are given by 


$$
\begin{gathered}
E_{r m}(\theta, \varphi)=E_{0} \cos (m \varphi)\left\{\begin{array}{cc}
P_{v}^{m}(\cos \theta), & 0 \leq \theta \leq \theta_{f}, \\
{\left[P_{v}^{m}(\cos \theta)+C_{m} Q_{v}^{m}(\cos \theta)\right],} & \theta_{f} \leq \theta \leq \theta_{p}{ }^{\prime}
\end{array}\right. \\
H_{\varphi m}(\theta, \varphi)=-E_{0} \frac{\sin \theta}{j \omega \mu_{0} a_{1}} \cos (m \varphi)\left\{\begin{array}{cc}
P_{v}^{\prime m}(\cos \theta), & 0 \leq \theta \leq \theta_{f} \\
{\left[P_{v}^{\prime m}(\cos \theta)+C_{m} Q_{v}^{\prime m}(\cos \theta)\right],} & \theta_{f} \leq \theta \leq \theta_{p}{ }^{\prime}
\end{array}\right.
\end{gathered}
$$

where $P_{v}^{m}$ and $Q_{v}^{m}$ are the associated Legendre functions of the first and second kind, respectively, while $C_{m}$ are unknown coefficients. Now, referring to Fig. 2, the circular patch is modeled as a $\Pi$-network $\left(g_{1}, g_{2}\right.$, and $\left.g_{3}\right) ; y_{f}$ is the wall admittance at $\theta=\theta_{f}^{-}, y_{p}$ is the wall admittance at the patch edge, and $I_{m}$ is the feed current corresponding to the $\mathrm{TM}_{m}$ mode excitation. The explicit expressions of the equivalent-circuit elements for the $\Pi$-network and the wall admittances at $\theta_{f}$ and $\theta_{p}$ are given in (Wong, 1999, p. 231-232).

Finally, having determined all the elements in the equivalent circuit, the input impedance of the antenna, seen by the probe feed at the $\mathrm{TM}_{11}$ mode, is given by

$$
Z_{i n}=\frac{d}{\pi}\left[y_{f}+g_{1}+\frac{g_{2}\left(g_{3}+y_{p}\right)}{g_{2}+g_{3}+y_{p}}\right]^{-1} .
$$

\section{A combined Legendre transform and T-matrix method for the analysis of multi-layered microstrips}

The techniques of Section 2 refer to the mathematical analysis of a single-layered spherical microstrip. In the present Section we summarize certain appropriate extensions of the aforementioned mathematical techniques in order to model the resonance and radiation characteristics of spherical microstrips composed of an arbitrary number of concentric spherical layers. For further details the reader is referred to (Valagiannopoulos \& Tsitsas, 2008a; Valagiannopoulos \& Tsitsas, 2008b). These extensions mainly refer to the suitable implementation of a T-matrix method, which handles effectively the effect of the dielectric layers above and below the PEC patch.

\subsection{Geometrical configuration of the multi-layered spherical microstrip}

The geometry of the under consideration multi-layered spherical microstrip is depicted in Fig. 3. A layered sphere of radius $a_{U+1}$ is divided by $U$ concentric spherical surfaces, defined by $r=a_{u}(u=1, \ldots, U)$, into $U+1$ layers $V_{u}(u=0, \ldots, U)$. The sphere's core $V_{0}\left(0 \leq r \leq a_{1}\right)$ is a perfect electric conductor. The layers $V_{u}$, defined by $a_{u} \leq r \leq a_{u+1}(u=1, \ldots, U)$, are filled with materials of complex dielectric permittivity $\varepsilon_{u}$ and wavenumber $k_{u}$. A PEC circular patch is printed on $r=a_{p}$ for $0 \leq \theta \leq \theta_{p}$ (between layers $V_{p-1}$ and $\left.V_{p}\right)$. The exterior $V_{U+1}\left(r>a_{U+1}\right)$ of the sphere is homogeneous with free-space dielectric permittivity $\varepsilon_{0}$, wavenumber $k_{0}$ and intrinsic impedance $\zeta_{0}$. The entire space is magnetically inert with permeability $\mu_{0}$. The feeding of the microstrip is not taken into account, since we focus on the inherent resonance and radiation properties of the microstrip structure and not the influence on them by an external excitation. However, we note that with the incorporation of certain appropriate modifications, a probe feeding of the microstrip may be also taken into account in the analysis (see for example the discussions in Chen et al., 1997). 


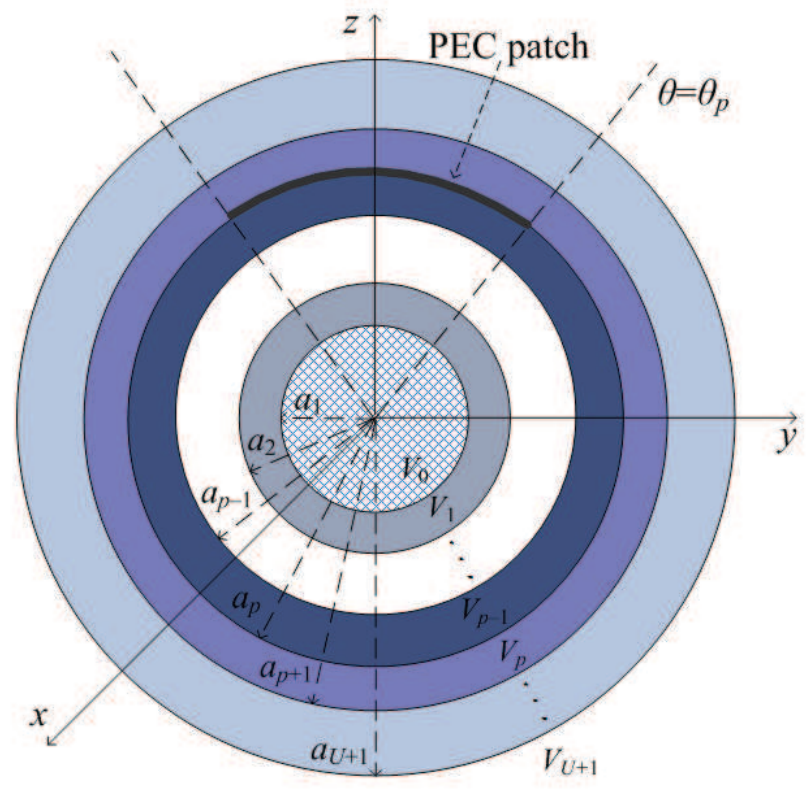

Fig. 3. The under consideration multi-layered spherical microstrip composed of a PEC spherical core of radius $a_{1}$ covered by an arbitrary number $U$ of dielectric layers; the circular PEC patch is located at $r=a_{p}$ and $\theta<\theta_{p}$ (between layers $V_{p-1}$ and $V_{p}$ ).

\subsection{T-matrix method}

The electric potential $\mathbf{A}_{u}$ and magnetic potential $\mathbf{F}_{u}$ in layer $V_{u}(u=1, \ldots, U+1)$, expressing the transverse magnetic $\mathrm{TM}^{r}$ and transverse electric $\mathrm{TE}^{r}$ modes respectively, admit the following expansions

$$
\begin{aligned}
\mathbf{A}_{u}(\mathbf{r}) & =\hat{\mathbf{r}} A_{u}(\mathbf{r})=\hat{\mathbf{r}} e^{j m \varphi} \sum_{n=|m|}^{+\infty}\left[\alpha_{u}(n) j_{n}\left(k_{u} r\right)+\beta_{u}(n) h_{n}\left(k_{u} r\right)\right] P_{n}^{m}(\cos \theta) \quad\left(a_{u} \leq r \leq a_{u+1}\right), \\
\mathbf{F}_{u}(\mathbf{r}) & =\hat{\mathbf{r}} F_{u}(\mathbf{r})=\hat{\mathbf{r}} e^{j m \varphi} \sum_{n=|m|}^{+\infty}\left[\gamma_{u}(n) j_{n}\left(k_{u} r\right)+\delta_{u}(n) h_{n}\left(k_{u} r\right)\right] P_{n}^{m}(\cos \theta) \quad\left(a_{u} \leq r \leq a_{u+1}\right),
\end{aligned}
$$

where $j_{n}$ and $h_{n}$ are the $n$-th order spherical Bessel and Hankel functions of the second kind, $P_{n} m$ is the associated Legendre function of the first kind of order $m$ and degree $n$ and $a_{u}, \beta_{u}$ $\gamma_{u}$, and $\delta_{u}$ are under determination coefficients. The electric and magnetic potentials in the exterior $V_{U+1}$ of the microstrip have the expansions (15) with $u=U+1$ and $a_{U+1}(n)=\gamma_{U+1}(n)=0$, valid for $r>a_{U+1}$, in order that the radiation condition is satisfied. The transverse electric and magnetic field components in layer $V_{u}$ are then readily expressed in terms of the corresponding potentials (see Eq. (2) of Valagiannopoulos \& Tsitsas, 2008a).

Next, since the spherical microstrip is non-entire with respect to $\theta$, we utilize the vector Legendre Transform, given by (1) and (2) (as analyzed in details in Section 2). The Legendre Transforms $\quad \tilde{\mathbf{E}}_{u}(r, n)=\left[\tilde{\mathbf{E}}_{u, \theta}(r, n), \tilde{\mathbf{E}}_{u, \varphi}(r, n)\right]^{T} \quad$ and $\quad \tilde{\mathbf{H}}_{u}(r, n)=\left[\tilde{\mathbf{H}}_{u, \theta}(r, n), \tilde{\mathbf{H}}_{u, \varphi}(r, n)\right]^{T} \quad$ of the 
transverse electric $\quad \mathbf{E}_{u}(r, n)=\left[\mathbf{E}_{u, \theta}(r, n), \mathbf{E}_{u, \varphi}(r, n)\right]^{T} \quad$ and magnetic

$\mathbf{H}_{u}(r, n)=\left[\mathbf{H}_{u, \theta}(r, n), \mathbf{H}_{u, \varphi}(r, n)\right]^{T}$ fields in layer $V_{u}$ are determined by means of (15) and (1b) and by imposing the orthogonality properties of the Legendre functions (Gradshteyn \& Ryzhik, 2000)

$$
\begin{gathered}
\tilde{\mathbf{E}}_{u}(r, n)=k_{0}\left[\begin{array}{c}
\sqrt{\varepsilon_{u}}\left(\gamma_{u}(n) j_{n}^{d}\left(k_{u} r\right)+\delta_{u}(n) h_{n}^{d}\left(k_{u} r\right)\right) \\
j \zeta_{0}\left(\alpha_{u}(n) j_{n}\left(k_{u} r\right)+\beta_{u}(n) h_{n}\left(k_{u} r\right)\right)
\end{array}\right], \\
\tilde{\mathbf{H}}_{u}(r, n)=k_{0}\left[\begin{array}{c}
\sqrt{\varepsilon_{u}}\left(\alpha_{u}(n) j_{n}^{d}\left(k_{u} r\right)+\beta_{u}(n) h_{n}^{d}\left(k_{u} r\right)\right) \\
\left(j \zeta_{0}\right)^{-1} \varepsilon_{u}\left(\gamma_{u}(n) j_{n}\left(k_{u} r\right)+\delta_{u}(n) h_{n}\left(k_{u} r\right)\right)
\end{array}\right],
\end{gathered}
$$

where $z j_{n}^{d}(z)=d\left(z j_{n}(z)\right) / d z$ and $z h_{n}^{d}(z)=d\left(z h_{n}(z)\right) / d z$. Notice that by using the Legendre Transform, the coefficient pairs $a_{u}, \beta_{u}$ and $\gamma_{u}, \delta_{u}$, corresponding to the $\mathrm{TM}^{r}$ and $\mathrm{TE}^{r}$ fields respectively, appear exclusively in only one component of the transformed vectors $\tilde{\mathbf{E}}_{u}, \tilde{\mathbf{H}}_{u}$, and thus a decoupling is achieved.

By imposing the vector Legendre Transform to the boundary conditions in the spatial domain, we obtain the following boundary conditions in the spectral domain

$$
\begin{gathered}
\tilde{\mathbf{E}}_{1}\left(a_{1}, n\right)=\mathbf{0}, \\
\tilde{\mathbf{E}}_{u}\left(a_{u}, n\right)-\tilde{\mathbf{E}}_{u-1}\left(a_{u}, n\right)=\mathbf{0} \quad(u=2, \ldots, U+1), \\
\tilde{\mathbf{H}}_{u}\left(a_{u}, n\right)-\tilde{\mathbf{H}}_{u-1}\left(a_{u}, n\right)=\mathbf{0} \quad(u=2, \ldots, U+1, u \neq p), \\
\tilde{\mathbf{J}}_{s}(n)=\left[\begin{array}{cc}
0 & -1 \\
1 & 0
\end{array}\right]\left(\tilde{\mathbf{H}}_{p}\left(a_{p}, n\right)-\tilde{\mathbf{H}}_{p-1}\left(a_{p}, n\right)\right),
\end{gathered}
$$

where $\mathbf{J}_{s}$ is the surface current distribution at $r=a_{p}$, due to the presence of the patch.

Now, we describe a T-matrix scheme, leading to the successive connection of the fields coefficients (connections between the fields coefficients of spherically layered media are analyzed in details in Tsitsas \& Athanasiadis, 2006). Specifically, successive applications of (17b) and (17c) imply the following transformations of the electric field coefficients in layer $V_{u-1}$ to those in $V_{u}$

$$
\begin{aligned}
& {\left[\begin{array}{l}
\alpha_{u}(n) \\
\beta_{u}(n)
\end{array}\right]=\overline{\overline{\mathbf{T}}}_{u}^{T M}(n)\left[\begin{array}{l}
\alpha_{u-1}(n) \\
\beta_{u-1}(n)
\end{array}\right] \quad(u=2, \ldots, U, u \neq p),} \\
& {\left[\begin{array}{l}
\gamma_{u}(n) \\
\delta_{u}(n)
\end{array}\right]=\overline{\overline{\mathbf{T}}}_{u}^{T E}(n)\left[\begin{array}{l}
\gamma_{u-1}(n) \\
\delta_{u-1}(n)
\end{array}\right] \quad(u=2, \ldots, U, u \neq p),}
\end{aligned}
$$

where the explicit expressions of the elements of the $2 \times 2$ transition matrices from $V_{u-1}$ to $V_{u}$, appearing above, are given in (Valagiannopoulos \& Tsitsas, 2008a). 
Furthermore, application of the boundary condition (17a) on the PEC core implies

$$
\begin{gathered}
{\left[\begin{array}{l}
\alpha_{1}(n) \\
\beta_{1}(n)
\end{array}\right]=\mathbf{t}_{1}^{T M}(n) \alpha_{1}(n),} \\
{\left[\begin{array}{l}
\gamma_{1}(n) \\
\delta_{1}(n)
\end{array}\right]=\mathbf{t}_{1}^{T E}(n) \gamma_{1}(n),}
\end{gathered}
$$

while application of $(17 \mathrm{~b})$ and $(17 \mathrm{c})$ for $u=U$ yields

$$
\begin{gathered}
{\left[\begin{array}{l}
\alpha_{U}(n) \\
\beta_{U}(n)
\end{array}\right]=\mathbf{t}_{U+1}^{T M}(n) \beta_{U+1}(n),} \\
{\left[\begin{array}{l}
\gamma_{U}(n) \\
\delta_{U}(n)
\end{array}\right]=\mathbf{t}_{U+1}^{T E}(n) \delta_{U+1}(n),}
\end{gathered}
$$

where the $2 \times 1$ vectors $\mathbf{t}_{1}^{T M}, \mathbf{t}_{1}^{T E}(n), \mathbf{t}_{U+1}^{T M}(n)$, and $\mathbf{t}_{U+1}^{T E}(n)$ are given in (Valagiannopoulos \& Tsitsas, 2008a).

Now, by applying successively the transformations (18) for $u=2, \ldots, p-1$ and using (19), we see that the field coefficients in layer $V_{p-1}$ are related to $a_{1}$ and $\gamma_{1}$ of $V_{1}$ by

$$
\begin{gathered}
{\left[\begin{array}{l}
\alpha_{p-1}(n) \\
\beta_{p-1}(n)
\end{array}\right]=\mathbf{t}_{-}^{T M}(n) \alpha_{1}(n),} \\
{\left[\begin{array}{l}
\gamma_{p-1}(n) \\
\delta_{p-1}(n)
\end{array}\right]=\mathbf{t}_{-}^{T E}(n) \gamma_{1}(n),}
\end{gathered}
$$

where

$$
\begin{aligned}
\mathbf{t}_{-}^{T M}(n) & =\left[\begin{array}{c}
f_{-}^{T M}(n) \\
g_{-}^{T M}(n)
\end{array}\right]=\overline{\overline{\mathbf{T}}}_{w-1}^{T M}(n) \overline{\overline{\mathbf{T}}}_{w-2}^{T M}(n) \cdots \overline{\overline{\mathbf{T}}}_{2}^{T M}(n) \mathbf{t}_{1}^{T M}(n), \\
\mathbf{t}_{-}^{T E}(n) & =\left[\begin{array}{l}
f_{-}^{T E}(n) \\
g_{-}^{T E}(n)
\end{array}\right]=\overline{\overline{\mathbf{T}}}_{w-1}^{T E}(n) \overline{\overline{\mathbf{T}}}_{w-2}^{T E}(n) \cdots \overline{\overline{\mathbf{T}}}_{2}^{T E}(n) \mathbf{t}_{1}^{T E}(n) .
\end{aligned}
$$

In a similar way by using (18) for $u=p+1, \ldots, U$ and (20), we obtain

$$
\begin{aligned}
& {\left[\begin{array}{c}
\alpha_{p}(n) \\
\beta_{p}(n)
\end{array}\right]=\mathbf{t}_{+}^{T M}(n) \beta_{U+1}(n),} \\
& {\left[\begin{array}{l}
\gamma_{p}(n) \\
\delta_{p}(n)
\end{array}\right]=\mathbf{t}_{+}^{T E}(n) \delta_{U+1}(n),}
\end{aligned}
$$

where 


$$
\begin{aligned}
& \mathbf{t}_{+}^{T M}(n)=\left[\begin{array}{l}
f_{+}^{T M}(n) \\
g_{+}^{T M}(n)
\end{array}\right]=\left[\overline{\overline{\mathbf{T}}}_{w+1}^{T M}(n)\right]^{-1}\left[\overline{\overline{\mathbf{T}}}_{w+2}^{T M}(n)\right]^{-1} \cdots\left[\overline{\overline{\mathbf{T}}}_{U}^{T M}(n)\right]^{-1} \mathbf{t}_{U+1}^{T M}(n), \\
& \mathbf{t}_{+}^{T E}(n)=\left[\begin{array}{l}
f_{+}^{T E}(n) \\
g_{+}^{T E}(n)
\end{array}\right]=\left[\overline{\overline{\mathbf{T}}}_{w+1}^{T E}(n)\right]^{-1}\left[\overline{\overline{\mathbf{T}}}_{w+2}^{T E}(n)\right]^{-1} \cdots\left[\overline{\overline{\mathbf{T}}}_{U}^{T E}(n)\right]^{-1} \mathbf{t}_{U+1}^{T E}(n) .
\end{aligned}
$$

The subscripts - in (21) and + in (23) indicate approach of the patch surface $r=a_{p}$ from the layers below and above respectively.

\subsection{Determination of the complex resonant frequencies}

By the continuity boundary condition (17b) for $u=p$, the tangential electric field of layer $V_{p-1}$ coincides with that of $V_{p}$; their common value will be denoted hereafter by $\tilde{\mathbf{E}}\left(a_{p}, n\right)$. Thus, by combining (17d), (21) and (23), we result to the following spectral equation for the surface current on the patch

$$
\tilde{\mathbf{E}}\left(\alpha_{p}, n\right)=\left[\overline{\overline{\mathbf{Y}}}_{+}(n)-\overline{\overline{\mathbf{Y}}}_{-}(n)\right]^{-1} \tilde{\mathbf{J}}_{s}(n),
$$

where

$$
\begin{gathered}
\overline{\overline{\mathbf{Y}}}_{+}(n)=j \frac{\sqrt{\varepsilon_{p}}}{\zeta_{0}}\left[\begin{array}{cc}
\frac{f_{+}^{T E}(n) j_{n}\left(x_{p}\right)+g_{+}^{T E}(n) h_{n}\left(x_{p}\right)}{f_{+}^{T E}(n) j_{n}^{d}\left(x_{p}\right)+g_{+}^{T E}(n) h_{n}^{d}\left(x_{p}\right)} & 0 \\
0 & -\frac{f_{+}^{T M}(n) j_{n}^{d}\left(x_{p}\right)+g_{+}^{T M}(n) h_{n}^{d}\left(x_{p}\right)}{f_{+}^{T M}(n) j_{n}\left(x_{p}\right)+g_{+}^{T M}(n) h_{n}\left(x_{p}\right)}
\end{array}\right], \\
\overline{\overline{\mathbf{Y}}}_{-}(n)=j \frac{\sqrt{\varepsilon_{p-1}}}{\zeta_{0}}\left[\begin{array}{cc}
\frac{f_{-}^{T E}(n) j_{n}\left(y_{p}\right)+g_{-}^{T E}(n) h_{n}\left(y_{p}\right)}{f_{-}^{T E}(n) j_{n}^{d}\left(y_{p}\right)+g_{-}^{T E}(n) h_{n}^{d}\left(y_{p}\right)} & 0 \\
0 & -\frac{f_{-}^{T M}(n) j_{n}^{d}\left(y_{p}\right)+g_{-}^{T M}(n) h_{n}^{d}\left(y_{p}\right)}{f_{-}^{T M}(n) j_{n}\left(y_{p}\right)+g_{-}^{T M}(n) h_{n}\left(y_{p}\right)}
\end{array}\right],
\end{gathered}
$$

while $x_{p}=k_{p} a_{p}, y_{p}=k_{p-1} a_{p}$.

Subsequently, we follow the general procedure, analyzed for the single-layered microstrip case in Sections 2.1 and 2.2. More precisely, the surface current density on the patch is expanded into a linear combination of the form (5) with respect to appropriate basis functions, which are specifically chosen by using the cavity model theory (Lo et al., 1979). Then, we apply a Galerkin's procedure for the computation of the linear combinations unknown weighting coefficients, finally resulting to the homogeneous linear system

$$
\overline{\overline{\mathbf{B}}}(\omega) \mathbf{c}=\mathbf{0}
$$

where

$$
B_{\mu v}(\omega)=\sum_{n=|m|}^{+\infty} S(n, m) \tilde{\mathbf{J}}_{s, \mu}^{*}(n)\left[\overline{\overline{\mathbf{Y}}}_{+}(n)-\overline{\overline{\mathbf{Y}}}_{-}(n)\right]^{-1} \tilde{\mathbf{J}}_{s, v}(n) \quad(\mu, v=1, \ldots, \mathrm{N})
$$


The possible complex resonant frequencies correspond to the non-trivial solutions of (27), namely to the values of the parameter $\omega$ for which the corresponding system matrix is singular. Hence, the complex resonant frequency $\omega_{0}=\operatorname{Re}\left(\omega_{0}\right)+j \operatorname{Im}\left(\omega_{0}\right)$ is the root of the determinant of (27); for further details see also the related discussion in Section 2.1.

It is worth to point-out that the elements of the matrix $\mathbf{B}$ in (27) are computed numerically by truncating the infinite sum. Thus, it is required to investigate the variation of the sufficient truncation order with respect to the microstrip characteristics, and in particular with respect to the angular extent $\theta_{p}$ of the patch. A typical convergence pattern of the resonant frequency of a three-layered spherical microstrip is depicted in Fig. 4 . It is evident that the required truncation order is large for $\theta_{p} \rightarrow 0$ and decreases rapidly as $\theta_{p}$ increases. The truncation order has also been tested against the variation of the other geometrical and physical parameters of the microstrip and it has been concluded that $\theta_{p}$ is the most significant parameter. For further details on the influence of the microstrip's parameters on the convergence pattern see (Valagiannopoulos \& Tsitsas, 2008a).

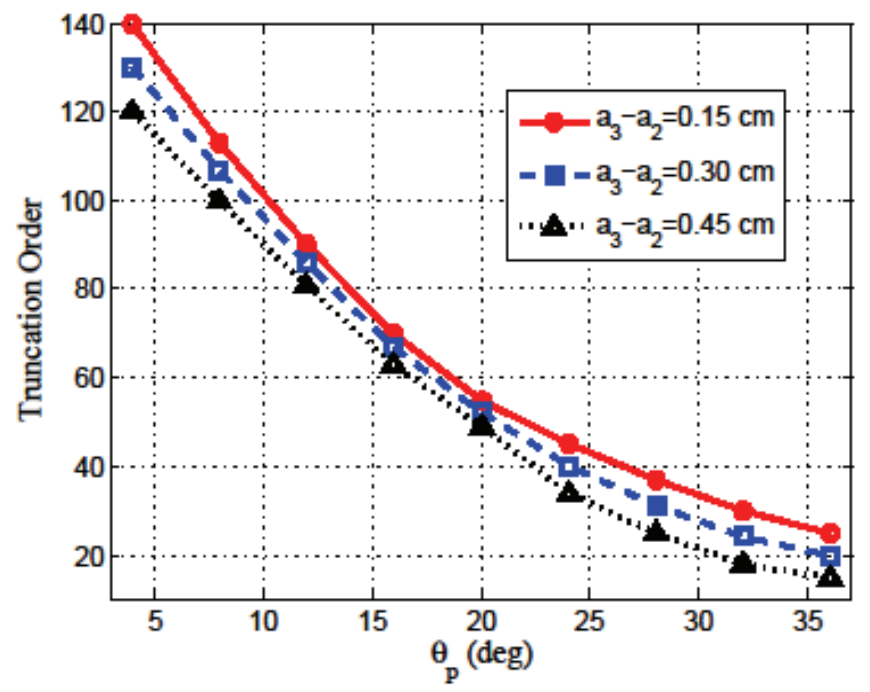

Fig. 4. Sufficient truncation order for convergence of the resonant frequency (as computed by solving the homogeneous linear system (27)) as a function of the angular extent $\theta_{p}$ of the patch for different superstrate thicknesses $a_{3}-a_{2}$ with $a_{1}=5 \mathrm{~cm}, a_{2}=5.15 \mathrm{~cm}, \varepsilon_{1}=\varepsilon_{2}=2.5 \varepsilon_{0}$ and $p=2$.

\subsection{Far-field radiation pattern}

The determined resonant frequency of (27) may be utilized for the computation of the farfield radiation pattern by means of the inverse Legendre Transform of (16). The patch current near the resonant frequency is well described by the cavity-model modes of (11). We consider only the fundamental $\mathrm{TM}_{11}$ mode and hence $\tilde{\mathbf{J}}_{s}(n)=\tilde{\mathbf{J}}_{s}^{T M_{11}}(n)$.

By following the procedure analyzed in (Valagiannopoulos \& Tsitsas, 2008a), we obtain the tangential electric field vector in the infinite exterior region $V_{U+1}$ 


$$
\begin{aligned}
\mathbf{E}_{U+1}(r, \theta) & =\left[\begin{array}{l}
\mathrm{E}_{\theta, U+1}(r, \theta) \\
\mathrm{E}_{\varphi, U+1}(r, \theta)
\end{array}\right] \\
= & k_{0}\left[\begin{array}{l}
\left.\sum_{n=1}^{+\infty}\left(\delta_{U+1}(n) h_{n}^{d}\left(k_{0} r\right) P_{n}^{\prime 1}(\cos \theta)+\zeta_{0} \beta_{U+1}(n) h_{n}\left(k_{0} r\right) \frac{P_{n}^{1}(\cos \theta)}{\sin \theta}\right)\right], \\
\sum_{n=1}^{+\infty}\left(j \delta_{U+1}(n) h_{n}^{d}\left(k_{0} r\right) \frac{P_{n}^{1}(\cos \theta)}{\sin \theta}+j \zeta_{0} \beta_{U+1}(n) h_{n}\left(k_{0} r\right) P_{n}^{\prime 1}(\cos \theta)\right)
\end{array}\right]
\end{aligned}
$$

where the coefficients $\delta_{U+1}, \beta_{U+1}$ are determined by means of the computed tangential electric field on the patch after combining (23) with (25).

Next, in order to obtain the far-field pattern expressions, we impose in (29) suitable asymptotic expansions of the spherical Hankel functions and their derivatives (Abramowitz \& Stegun, 1972) and finally result to

$$
\mathbf{E}_{U+1}(r, \theta) \sim h_{0}\left(k_{0} r\right)\left[\begin{array}{c}
P_{\theta}(\theta) \\
P_{\varphi}(\theta)
\end{array}\right], k_{0} r \rightarrow+\infty,
$$

where the far-field pattern components are given by

$$
\left[\begin{array}{c}
P_{\theta}(\theta) \\
P_{\varphi}(\theta)
\end{array}\right]=e^{-j \frac{\pi}{4}} k_{0}\left[\begin{array}{l}
\sum_{n=1}^{+\infty} j^{n}\left(-j \delta_{U+1}(n) P_{n}^{\prime 1}(\cos \theta)+\zeta_{0} \beta_{U+1}(n) \frac{P_{n}^{1}(\cos \theta)}{\sin \theta}\right) \\
\sum_{n=1}^{+\infty} j^{n}\left(\delta_{U+1}(n) \frac{P_{n}^{1}(\cos \theta)}{\sin \theta}+j \zeta_{0} \beta_{U+1}(n) P_{n}^{\prime 1}(\cos \theta)\right)
\end{array}\right] .
$$

\section{Applications}

In this Section various interesting multi-layered spherical microstrip configurations will be presented and their operational characteristics will be briefly discussed. The complex resonant frequencies of these configurations as well as their far-field radiation patterns will be computed by the combined vector Legendre Transform and T-matrix technique, outlined in Section 3. For simplicity, we take into account hereinafter only the fundamental $\mathrm{TM}_{11}$ mode, in order to adopt a qualitative approach for the investigation of certain novel spherical microstrip configurations. However, by following the general analysis of Section 3, the respective numerical results corresponding to higher order modes can be also readily computed.

\subsection{Zero refractive index metamaterial coating}

The merits of using zero refractive index materials as substrates in planar antennas have been highlighted in (Wu et al. 2005; Caloz \& Itoh, 2006). Such materials are usually realized by periodic collections of rods and rings (Grzegorczyk et al. 2005). Particularly, in (Pendry et al. 1998) it was shown that by using a metallic mesh of thin wires, a plasma-like metamaterial medium is obtained, which is characterized by zero effective permittivity (and hence zero refractive index) at the plasma frequency. Zero index materials possess (according to Snell's law) the property of aligning the traveling rays from inside such a medium to free space, regardless of the angle of incidence, hence achieving high directivity by controlling the direction of emission (see Fig. 1 of Wu et al. 2005). 
The spherical analog of such an emitting antenna does not lead to equally interesting results, since its operation might be expected to be nearly omnidirectional. However, the same configuration, utilized as a receiving antenna, could be potentially more promising. More precisely, suppose a spherical shell of zero refractive index enclosing the whole spherical microstrip. Then, by local application of Snell's law (similarly to the planar case), one would expect that all the ingoing rays traveling inside the zero index medium would enter the

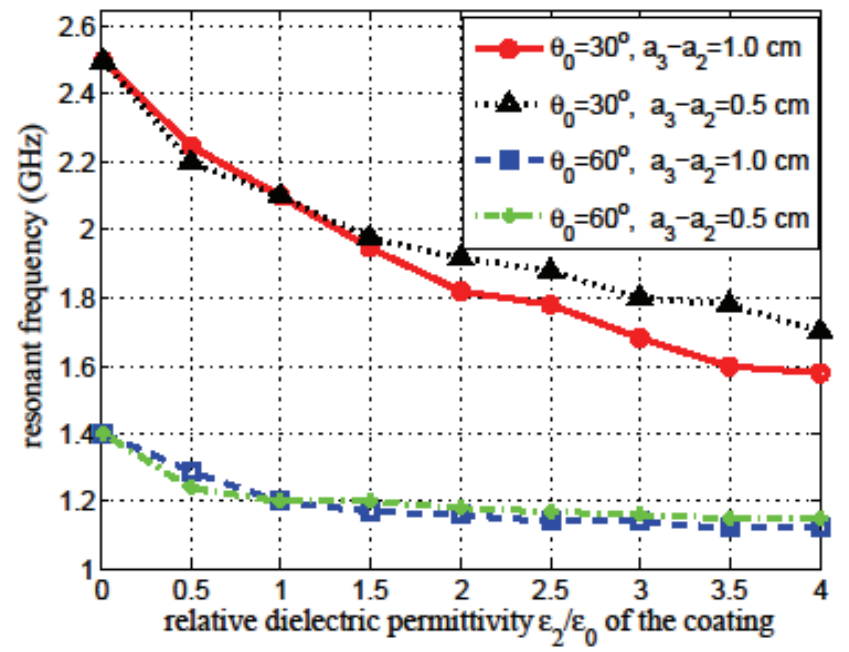

(a)

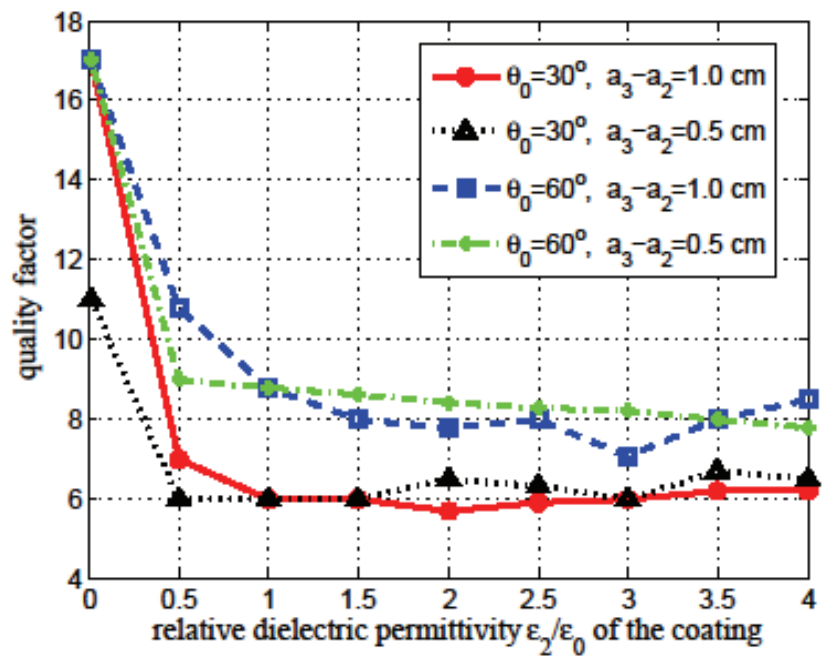

(b)

Fig. 5. Variations of (a) resonant frequency and (b) quality factor with respect to the coating's relative permittivity $\varepsilon_{2} / \varepsilon_{0}$ for different coating's thicknesses $a_{3}-a_{2}$ and angular patch extents $\theta_{p}$ in the case of a 3-layered microstrip with $a_{1}=6 \mathrm{~cm}, a_{2}=7 \mathrm{~cm}, p=2$ and $\varepsilon_{1}=\varepsilon_{0}$. 
microstrip aligned perpendicular to the local tangential planes. Thus, the rays arrive with minimum path losses either on the patch or on the surface of the PEC sphere, therefore improving the receiving procedure. A representative sketch for the clarification of this physical situation is depicted in (Fig. 5 of Valagiannopoulos \& Tsitsas, 2008a).

The preceding considerations motivate the investigation of the resonant properties of a spherical microstrip surrounded by a zero refractive index material. To this end, we consider a 3-layered microstrip with an airgap between the PEC sphere and the patch and a material with varying dielectric permittivity and thickness between the patch and the free space. Figs. $5 \mathrm{a}$ and $5 \mathrm{~b}$ show the variation of the resonant frequency and quality factor of the aforementioned microstrip with respect to the coating's dielectric permittivity for two different coating's thicknesses $a_{3}-a_{2}$ and two different angular extents $\theta_{p}$ of the patch. The resonant frequency decreases and the quality factor increases with increasing angular extent $\theta_{p}$ for fixed coating's thickness and dielectric permittivity. Especially, $Q$ attains a global maximum value for $\varepsilon_{2} \rightarrow 0$, demonstrating that such a microstrip also possesses a high quality factor, when a material with nearly zero refractive index is utilized as coating. Furthermore, by Fig. 5a it is evident that for fixed $\theta_{p}$ the influence of the coating's thickness on the resonant frequency becomes weaker as its dielectric permittivity $\varepsilon_{2}$ decreases.

\subsection{Amplifying excitation layer}

The feeding of the spherical microstrip is usually modeled as a source located between the PEC core and the patch (Chen et al., 1997; Sipus et al., 2008; Khamas, 2009). A common type of source utilized in such cases is an axial probe connecting the two PEC surfaces. However, the antenna's operation is strongly dependent on the position of the feeding.

To this direction, a new type of $\varphi$ - and $\theta$ - entire excitation, not affecting considerably the inherent resonance and radiation properties of the antenna, was proposed in (Valagiannopoulos \& Tsitsas, 2008a). This excitation is an active (plasma) layer, located between core and patch, and filled with material possessing dielectric permittivity of positive imaginary part. Note that the plasma behavior of the material should be achieved close to the microstrip's resonant frequency.

In particular, a 4-layered microstrip with two airgaps surrounding the active layer was considered with the amplifying capability being controllable via both the layer's thickness $a_{3}-a_{2}$ as well as its dielectric permittivity $\varepsilon_{2}=\operatorname{Re}\left[\varepsilon_{2}\right]+j \operatorname{Im}\left[\varepsilon_{2}\right]$. Figs $6 \mathrm{a}$ and $6 \mathrm{~b}$ depict the variation of the resonant frequency and the quality factor with respect to the active layer's thickness for different imaginary parts of its permittivity. The resonant frequency seems to be rather insensitive to the increase of $\operatorname{Im}\left[\varepsilon_{2}\right]$, indicating a rather weak dependence of the resonant frequency on the amplifying capability of the active layer. As far as the quality factor is concerned, it is indeed an increasing function of both $a_{3}-a_{2}$ and $\operatorname{Im}\left[\varepsilon_{2}\right]$. Specifically, in applications demanding large values of $Q$, this configuration may be used with $a_{3} \rightarrow a_{4}$ and $\operatorname{Im}\left[\varepsilon_{2}\right]$ suitably large.

\subsection{Shifted Luneburg lens material}

In the literature are usually examined microstrips with a fixed small number of concentric layers (Tam \& Luk, 1991; Wong et al., 1993a; Wong \& Chen, 1993). However, it is particularly interesting to investigate also the case of the coating's region $a_{1}<r<a_{U+1}$ having continuously varying dielectric permittivity with radial dependence because such a configuration would offer more degrees of freedom to the designer in order to achieve the 


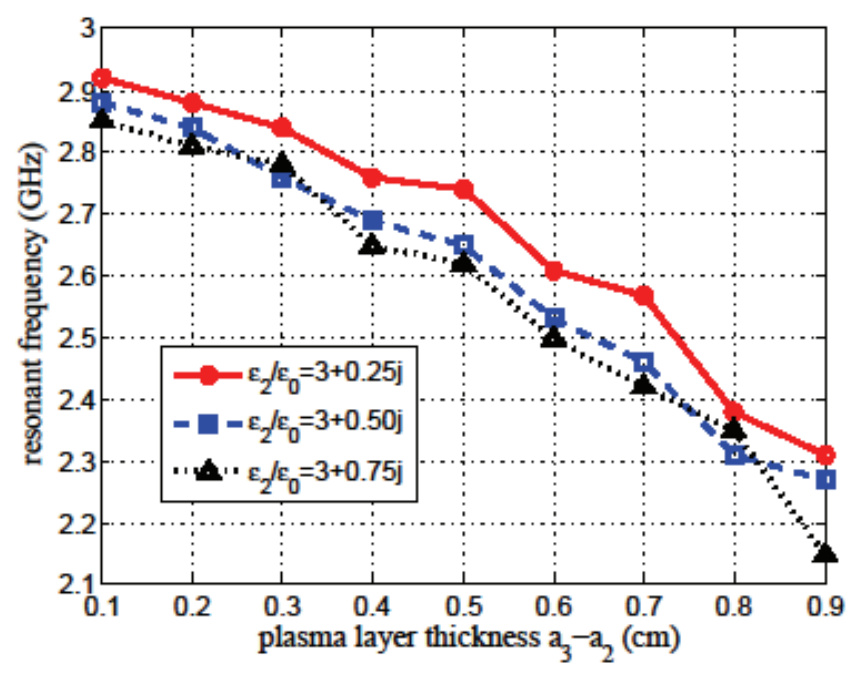

(a)

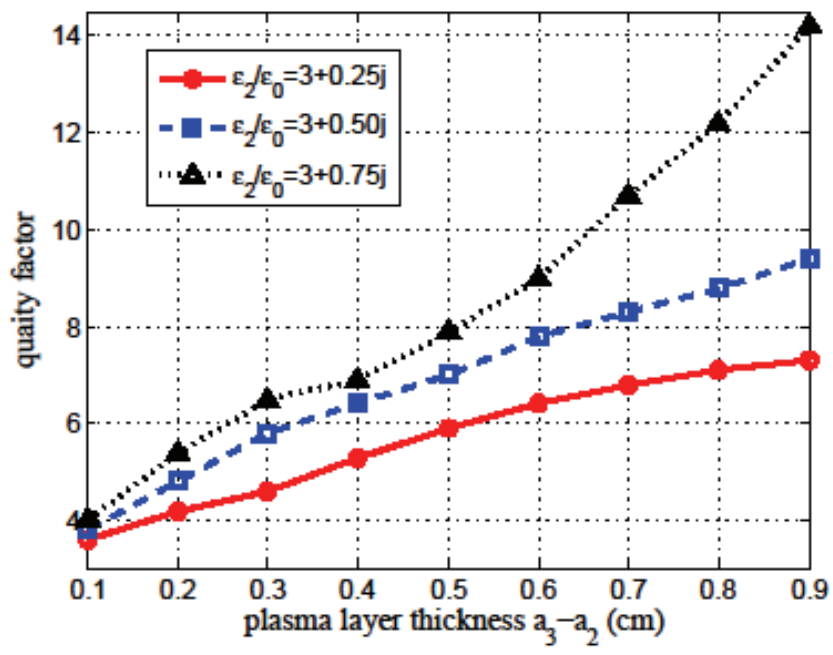

(b)

Fig. 6. (a) Resonant frequency and (b) quality factor as functions of the active (plasma) layer's thickness for different imaginary parts of its dielectric permittivity for a 4-layered microstrip with $a_{1}=6 \mathrm{~cm}, a_{2}=6.5 \mathrm{~cm}, a_{4}=7.5 \mathrm{~cm}, p=4, \theta_{p}=15^{\circ}$, and $\varepsilon_{1}=\varepsilon_{3}=\varepsilon_{0}$.

required operational characteristics. Since the method, summarized in Section 3, is suitable for handling efficiently microstrips with arbitrary number $U$ of spherical layers, a continuous distribution of the radial function of the dielectric permittivity may be effectively treated by the corresponding step approximation. 
In (Valagiannopoulos \& Tsitsas, 2008a) a spherical microstrip with discrete shiftedLuneburg coating was investigated. More precisely, since the microstrip is composed of a PEC core, its coating is assumed to obey a continuous distribution with radial dependence following a shifted Luneburg lens law (Luneburg, 1941)

$$
\varepsilon(r)=2-\left(\frac{r-a_{1}}{a_{U+1}-a_{1}}\right)^{2} \quad\left(a_{1}<r<a_{U+1}\right) .
$$

This continuous distribution is approximated by the analyzed model of $U$ spherical layers. The patch is still located inside the coating on $r=a_{p}$.

Typical variations of the resonant frequency and the quality factor as functions of the patch location $a_{p}$ inside the discrete Luneburg coating for different angular extents $\theta_{p}$ of the patch are depicted in Fig. 7 of (Valagiannopoulos \& Tsitsas, 2008a). The resonant frequency decreases with increasing $\theta_{p}$ for fixed $a_{p}$ and with increasing $a_{p}$ for fixed $\theta_{p}$. On the other hand, the quality factor decreases rapidly as the patch gets distant from the PEC sphere for fixed $\theta_{p}$ and increases with $\theta_{p}$ for fixed $a_{p}$ (as opposed to the respective behavior of the resonant frequency for increasing $\theta_{p}$ ). The values of the quality factor become extremely large as the patch approaches the PEC sphere, a fact which may be exploited appropriately in various applications.

Finally, regarding the electric far-field pattern components $P_{\theta}(\theta)$ and $P_{\varphi}(\theta)$, which are computed by means of (31), Figs $7 \mathrm{a}$ and $7 \mathrm{~b}$ depict the variation of the normalized components $\left|P_{\theta}(\theta)\right|$ and $\left|P_{\varphi}(\theta)\right|$ for three different patch locations $a_{p}$ inside the Luneburg coating with fixed patch angular extent $\theta_{p}=30^{\circ}$. For all three patch locations the curves of $\left|P_{\theta}(\theta)\right|$ attain three local maxima at $00^{\circ}, 95^{\circ}$, and $180^{\circ}$, and two local minima at $50^{\circ}$, and $135^{\circ}$. On the other hand, the curves of $\left|P_{\varphi}(\theta)\right|$ attain two local maxima at $0^{\circ}$, and $180^{\circ}$, and one minimum at 110 . The locations of these maxima and minima do not seem to be strongly dependent on the patch location. However, their values increase as $a_{p}$ increases, namely as patch gets distant from the PEC sphere $V_{0}$ and gets closer to the free-space region $V_{U+1}$.

\section{Conclusions}

Spherical microstrip antennas have certain important advantages, which have been meticulously highlighted in the literature. Among the mathematical methods, which have been developed for analyzing the behavior of a spherical microstrip, the most widely used and effective ones are the full-wave approach, the cavity model analysis, and the generalized transmission-line model. A brief overview of each of these methods has been given. On the other hand, we summarized the basic features of an efficient method which combines the vector Legendre Transform with the T-matrix method and is utilized to analyze and model the resonance and radiation phenomena by a multi-layered spherical microstrip, whose circular metallic patch is located inside a coating, composed of an arbitrary number of concentric spherical substrate and superstrate layers. Finally, certain potential applications of multi-layered spherical microstrips were pointed out, where emphasis was given on the variation of the resonant frequency with respect to the microstrip's physical and geometrical characteristics as well as on the conditions under which high values of the quality factor may be achieved. 


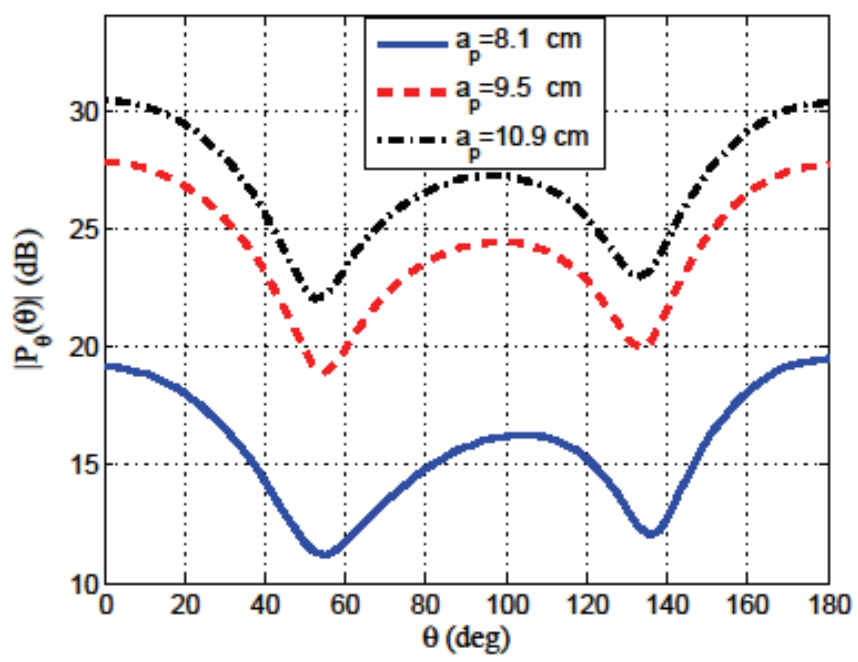

(a)

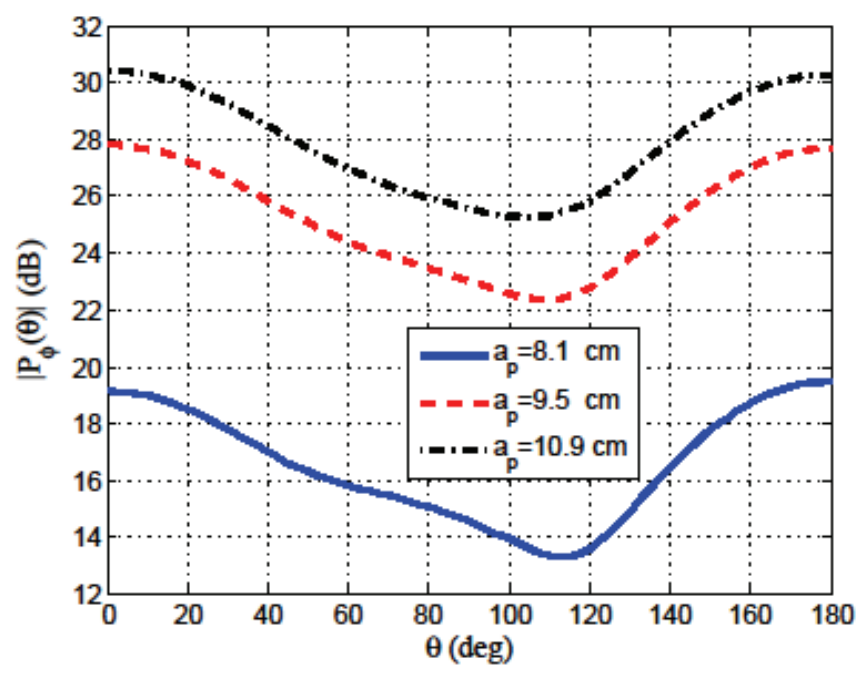

(b)

Fig. 7. Variation of the normalized electric far-field components $\left|P_{\theta}(\theta)\right|$ and $\left|P_{\varphi}(\theta)\right|$ for three different patch locations $a_{p}$ with fixed patch angular extent $\theta_{p}=30^{\circ}$, inside a discrete Luneburg coating with constant parameters $a_{1}=8 \mathrm{~cm}, a_{21}=11 \mathrm{~cm}, U=20$. 


\section{References}

Abramowitz M. \& Stegun I. (1972). Handbook of Mathematical Functions, Dover, New York

Ali S. M., Chew W. C. \& Kong J. A. (1982). Vector Hankel Transform Analysis of AnnularRing Microstrip Antenna. IEEE Trans. Antennas Propag., Vol. AP-30, No. 4, pp. 637644

Bhattacharyya A. K. \& Garg R. (1985). Generalised transmission line model for microstrip patches. IEE Proc. pt. H., Vol. 132, pp. 93-98.

Caloz C. \& Itoh T. (2006). Electromagnetic Metamaterials: Transmission Line Theory and Microwave, John Wiley \& Sons, New Jersey

Chen H. T. \& Wong K. L. (1994). Analysis of probe-fed spherical circular microstrip antennas using cavity-model theory. Microwave Opt. Technol. Lett., Vol. 25, pp. 309312

Chen H.-T., Chen H.-D \& Cheng Y.-T. (1997). Full-Wave Analysis of the Annular-Ring Loaded Spherical-Circular Microstrip Antenna. IEEE Trans. Antennas Propag., Vol. 45 , No. 11 , pp. $1581-1583$

Chew W. C. (1995). Waves and Fields in Inhomogeneous Media, IEEE Press, New York

Giang T. V. B., Thiel M. \& Dreher A. (2005). A Unified Approach to the Analysis of Radial Waveguides, Dielectric Resonators, and Microstrip Antennas on Spherical Multilayer Structures. IEEE Trans. Microw. Theory Tech., Vol. 53, No. 1, pp. 404-409

Gradshteyn I. S. \& Ryzhik I. M. (2000). Table of Integrals, Series, and Products, Academic Press, San Diego

Grzegorczyk T. M., Moss C. D., Lu J., Chen X., Pacheco J. Jr. \& Kong J. A. (2005). Properties of Left-Handed Metamaterials: Transmission, Backward Phase, Negative Refraction, and Focusing. IEEE Trans. Microw. Theory Tech., Vol. 53, No. 9, pp. 29562967

Ke B., \& Kishk A. A. (1991). Analysis of spherical circular microstrip antennas. IEE Proc. pt. H, Vol. 138, pp. 542-548

Khamas S. K. (2009). Electromagnetic Radiation by Antennas of Arbitrary Shape in a Layered Spherical Media. IEEE Trans. Antennas Propag., Vol. 57, No. 12, pp. 38273834

Kim J. \& Rahmat-Samii Y. (2004). Implanted Antennas Inside a Human Body: Simulations, Designs, and Characterizations. IEEE Trans. Microw. Theory Tech., Vol. 52, No. 8, pp. 1934-1943

Kishk A. A. (1993). Analysis of spherical annular-ring microstrip antennas. IEEE Trans. Antennas Propagat., Vol. 41, pp. 338-343

Liang C. S., Streater D. A., Jin J.-M., Dunn E., \& Rozendal T. (2005). A quantitative study of Luneberg-lens reflectors. IEEE Antenna Propag. Mag., Vol. 47, No. 2, pp. 30-41

Lo Y. T., Solomon D., \& Richards W. F. (1979). Theory and Experiment on Microstrip Antennas. IEEE Trans. Antennas Propag., Vol. AP-27, No. 2, pp. 137-145

Luk K. M. \& Tam W. Y. (1991). Patch antennas on spherical body. Proc. Inst. Elect. Eng., pt. H, Vol. 138, pp. 103-108

Luneburg R. K. (1941). The Mathematical Theory of Optics, Brown Univ. Press: Providence, RI.

Nie Z., Chew W. C. \& Lo Y. T. (1990). Analysis of the Annular-Ring-Loaded Circular-Disk Microstrip Antenna. IEEE Trans. Antennas Propag., Vol. 38, No. 6, pp. 806-813 
Nikolic N., Kot J. S., \& Vinogradov S. (2007). Scattering by a Luneberg lens partially covered by a metallic cap. J. of Electromagn. Waves and Appl., Vol. 21, No. 4, pp. 549-563

Pendry J. B., Holden A. J., Robbins D. J. \& Stewart W. J. (1998). Low frequency plasmons in thin-wire structures. J. Physics-Condensed Matter, Vol. 10, pp. 4785-4809

Pozar D. \& Schaubert D. (1995). Microstrip Antennas: The Analysis and Design of Microstrip Antennas and Arrays, Wiley-IEEE Press: New York

Pues H. \& Van de Capelle A. (1984). Accurate transmission-line model for the rectangular microstrip antenna. IEE Proc. pt. H, Vol. 131, pp. 334-340

Ribero J.-M., Damiano J.-P. \& Pirinoli P. (1999). Analysis and synthesis of spherical circular and annular-ring microstrip structures using a new algebraic tool. Microw. and Opt. Tech. Lett., Vol. 20, No. 4, pp. 274-280

Sakurai H., Hashidate T., Ohki M., Motojima K., \& Kozaki S. (1998). Electromagnetic scattering by the Luneberg lens with reflecting cap. IEEE Trans. Electromagn. Compat., Vol. 40, No. 2, pp. 94-96

Sipus Z., Burum N. \& Bartolic J. (2003). Analysis of rectangular microstrip patch antennas on spherical structures. Microw. and Opt. Tech. Lett., Vol. 36, No. 4, pp. 276-280

Sipus Z., Skokic S., Bosiljevac M. \& Burum N. (2008). Study of Mutual Coupling Between Circular Stacked-Patch Antennas on a Sphere. IEEE Trans. Antennas Propag., Vol. 56, No. 7, pp. 1834-1844

Tam W. Y. \& Luk K. M. (1991). Resonance in spherical-circular microstrip structure. IEEE Trans. Microwave Theory Tech., Vol. 39, pp. 700-704

Tsalamengas J. L., Uzunoglu N. K. \& Alexopoulos N. G. (1985). Propagation Characteristics of a Microstrip Line Printed on a General Anisotropic Substrate. IEEE Trans. Microw. Theory Tech., Vol. MTT-33, No. 10, pp. 941-945

Tsitsas N. L. \& Athanasiadis C. (2006). On the scattering of spherical electromagnetic waves by a layered sphere. Quart. J. Mech. Appl. Math., Vol. 59, No. 1, pp. 55-74

Uwaro, T. \& Itoh, T. (1989). Chapter 5, In: Numerical Techniques for Microwave and MillimeterWave Passive Structures, Itoh T., (Ed.), John Wiley \& Sons, New York

Valagiannopoulos C. A. \& Tsitsas N. L. (2008a). On the Resonance and Radiation Characteristics of Multi-Layered Spherical Microstrip Antennas. Electromagnetics, Vol. 28, pp. 243-264

Valagiannopoulos C. A. \& Tsitsas N. L. (2008b). Complex resonant frequencies of multilayered spherical microstrip antennas, Proceedings of $12^{\text {th }}$ International Conference on Mathematical Methods in Electromagnetic Theory, 2008 (MMET 2008), pp. 213-215, Odessa, Ukraine, Jun. 2008

Wong, K.-L. (1999). Design of Nonplanar Microstrip Antennas and Transmission Lines, Wiley, New York

Wong K.-L. \& Chen H.-T. (1993). Resonance in a Spherical-Circular Microstrip Structure with an Airgap. IEEE Trans. Microw. Theory Tech., Vol. 41, No. 8, pp. 1466-1468

Wong K.-L., Hsiao S.-F. \& Chen H.-T. (1993a). Resonance and Radiation of a SuperstrateLoaded Spherical-Circular Microstrip Patch Antenna. IEEE Trans. Antennas Propag., Vol. 41, No. 5, pp. 686-690 
Wong K.-L., Row J.-S., Kuo C.-W. \& Huang K.-C. (1993b). Resonance of a Rectangular Microstrip Patch on a Uniaxial Substrate. IEEE Trans. Microw. Theory Tech., Vol. 41, No. 4, pp. 698-701

Wu B.-I., Wang W., Pacheco J., Chen X., Grzegorczyk T. \& Kong J. A. (2005). A study of using metamaterials as antenna substrate to enhance gain. Progress in Electromagnetic Research, Vol. PIER 51, pp. 295-328 


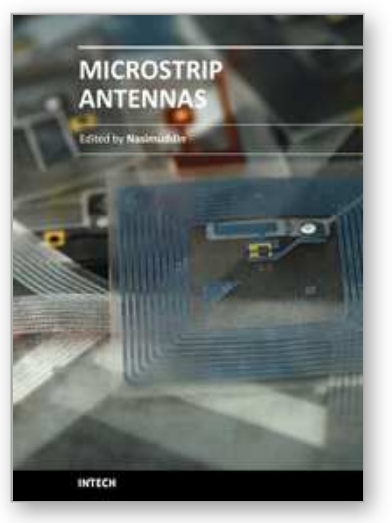

\author{
Microstrip Antennas \\ Edited by Prof. Nasimuddin Nasimuddin
}

ISBN 978-953-307-247-0

Hard cover, 540 pages

Publisher InTech

Published online 04, April, 2011

Published in print edition April, 2011

In the last 40 years, the microstrip antenna has been developed for many communication systems such as radars, sensors, wireless, satellite, broadcasting, ultra-wideband, radio frequency identifications (RFIDs), reader devices etc. The progress in modern wireless communication systems has dramatically increased the demand for microstrip antennas. In this book some recent advances in microstrip antennas are presented.

\title{
How to reference
}

In order to correctly reference this scholarly work, feel free to copy and paste the following:

Nikolaos L. Tsitsas and Constantinos A. Valagiannopoulos (2011). Mathematical Modeling of Spherical Microstrip Antennas and Applications, Microstrip Antennas, Prof. Nasimuddin Nasimuddin (Ed.), ISBN: 978953-307-247-0, InTech, Available from: http://www.intechopen.com/books/microstrip-antennas/mathematicalmodeling-of-spherical-microstrip-antennas-and-applications

\section{INTECH}

open science | open minds

\author{
InTech Europe \\ University Campus STeP Ri \\ Slavka Krautzeka 83/A \\ 51000 Rijeka, Croatia \\ Phone: +385 (51) 770447 \\ Fax: +385 (51) 686166 \\ www.intechopen.com
}

\author{
InTech China \\ Unit 405, Office Block, Hotel Equatorial Shanghai \\ No.65, Yan An Road (West), Shanghai, 200040, China \\ 中国上海市延安西路65号上海国际贵都大饭店办公楼405单元 \\ Phone: +86-21-62489820 \\ Fax: +86-21-62489821
}


(C) 2011 The Author(s). Licensee IntechOpen. This chapter is distributed under the terms of the Creative Commons Attribution-NonCommercialShareAlike-3.0 License, which permits use, distribution and reproduction for non-commercial purposes, provided the original is properly cited and derivative works building on this content are distributed under the same license. 